\title{
Sugar metabolism and the plant target of rapamycin kinase: a sweet operaTOR?
}

\section{Thomas Dobrenel ${ }^{1}$, Chloé Marchive ${ }^{1}$, Marianne Azzopardi ${ }^{1}$, Gilles Clément ${ }^{1}$, Manon Moreau ${ }^{1,2}$, Rodnay Sormani ${ }^{1}$, Christophe Robaglia ${ }^{2}$ and Christian Meyer ${ }^{1}{ }^{*}$}

\author{
1 Institut Jean-Pierre Bourgin, UMR 1318 INRA AgroParisTech, Saclay Plant Sciences, Versailles, France \\ ${ }^{2}$ Laboratoire de Génétique et Biophysique des Plantes, UMR 7265, DSV, IBEB, SBVME, CEA, CNRS, Faculté des Sciences de Luminy, Aix Marseille Université, \\ Marseille, France
}

\section{Edited by:}

Sjef Smeekens, Utrecht University, Netherlands

Reviewed by:

Sjef Smeekens, Utrecht University, Netherlands

Patrick Giavalisco, Max Planck Institute of Molecular Plant

Physiology, Germany

\section{*Correspondence:}

Christian Meyer, Institut Jean-Pierre Bourgin, UMR 1318 INRA

AgroParisTech, Institut National de la Recherche Agronomique Versailles, 78026 Versailles Cedex, France.

e-mail: cmeyer@versailles.inra.fr
In eukaryotes, the ubiquitous TOR (target of rapamycin) kinase complexes have emerged as central regulators of cell growth and metabolism. The plant TOR complex 1 (TORC1), that contains evolutionary conserved protein partners, has been shown to be implicated in various aspects of $\mathrm{C}$ metabolism. Indeed Arabidopsis lines affected in the expression of TORC1 components show profound perturbations in the metabolism of several sugars, including sucrose, starch, and raffinose. Metabolite profiling experiments coupled to transcriptomic analyses of lines affected in TORC1 expression also reveal a wider deregulation of primary metabolism. Moreover recent data suggest that the kinase activity of TORC1, which controls biological outputs like mRNA translation or autophagy, is directly regulated by soluble sugars.

Keywords: target of rapamycin, starch, raffinose, myo-inositol-1-phosphate synthase, TOR serine-threonine kinases

\section{INTRODUCTION}

The adjustment of primary metabolism to environmental conditions and to the availability of energy and nutrients is of primary importance to maintain cell homeostasis. Plants, like other eukaryotic organisms, have evolved to make an optimal use of nutrients and to adapt to nutritional deficiencies. This implies that plants have the ability to monitor the amount of available nutrients and energy and to adapt their transcriptional, translational, and metabolic responses to this information. In animals, in which cells are continuously maintained in a rather buffered and uniform supply of nutrients, this regulation of metabolic activity and cell growth at the cellular level is mainly driven by growth factors and hormones. For plants, nutrients provide not only the food for growth but also the signals for growth. Indeed nutrients serve both as the resources by which the cell increases mass and generates energy and as the signals controlling the metabolic and developmental programs which optimize survival under particular nutritional states.

Furthermore, plants experience rapid, sudden, and often long changes from optimal growth conditions and they must be able to both monitor precisely these changes and to trigger counter-measures ensuring survival and adaptation while maintaining growth and biomass production. In plants, like in other eukaryotes, the signaling pathway involving the TOR (target of rapamycin) protein kinase has emerged as an evolutionary conserved and critical link between external cues and metabolic and growth adaptations (see Wullschleger et al., 2006; Ma and Blenis, 2009; Loewith and Hall, 2011; Laplante and Sabatini, 2012; Cornu et al., 2013 for general reviews and Dobrenel et al., 2011; John et al., 2011; Robaglia et al., 2012 for reviews on the plant TOR signaling pathway).
Target of rapamycin was identified 20 years ago in yeast in a screen for mutations conferring resistance to rapamycin, an antibiotic that stops growth and induces a shift to the G0 quiescent stage (Heitman et al., 1991). It was later shown that rapamycin inhibits TOR by triggering the formation of an artificial complex between the TOR FRB (FKBP12-rapamycin binding)domain and the small FKBP12 protein (Wullschleger et al., 2006). Rapamycin treatment inhibits some of the TOR-linked activities and results, in yeast and animal cells, in the accumulation of the storage compound glycogen, in translation decrease and in the induction of autophagy (Schmelzle et al., 2004; Rohde et al., 2008; Broach, 2012; Cornu et al., 2013). These changes also occur in nutrient-starved cells (Rohde et al., 2008; Broach, 2012), which suggests that TOR is one of the main components of the transduction chain linking nutrient signaling to cellular adaptations. Indeed a wealth of studies, both in yeast and in animals, have clearly established that the TOR kinase is activated by external signals like the availability of amino acids or the presence of hormones, and then controls a myriad of biological outputs including transcription of RNA, translation, ribosome biogenesis, translocation of regulatory proteins, autophagy, and storage of reserve compounds (see above reviews). This review will mainly focus on the cross-talk between the conserved plant TOR kinase signaling pathway and $\mathrm{C}$ metabolism with a particular emphasis on C storage compounds.

\section{THE TOR KINASE}

The large TOR kinase associates in high molecular mass complexes with other conserved protein partners (Wullschleger et al., 2006; Loewith and Hall, 2011; Wang and Proud, 2011). In yeast and animals, the TOR kinase functions in two distinct multiprotein complexes named TOR complex 1 (TORC1) and TOR complex 2 
(TORC2). The rapamycin-sensitive TORC1 contains three major proteins (TOR, KOG1/RAPTOR, and LST8/GbetaL), which are also found in plants (Menand et al., 2002; Anderson et al., 2005; Deprost et al., 2005; Mahfouz et al., 2006; Moreau et al., 2012) and is thought to mainly regulate metabolism, mRNA translation, and autophagy (Wang and Proud, 2011). The TOR/RAPTOR and TOR/LST8 interactions have also been established in plants (Mahfouz et al., 2006; Díaz-Troya et al., 2008; Moreau et al., 2012). The TORC2 complex contains LST8/GbetaL with specific proteins like AVO3/RICTOR and AVO1/SIN1 (Wullschleger et al., 2006). The existence of the TORC2 complex in plants has not been proven so far but it may represent a more recent addition to the TOR signaling pathway.

It was previously thought that rapamycin, even at important doses, does not affect plant growth. Indeed plant FKBP12 proteins carry mutations that would preclude the formation of a TOR-rapamycin-FKBP12 ternary complex (Xu et al., 1998; Menand et al., 2002; Mahfouz et al., 2006; Sormani et al., 2007). Accordingly no interactions were detected between Arabidopsis TOR and FKBP12 proteins using two-hybrid techniques (Mahfouz et al., 2006; Sormani et al., 2007). However, the Arabidopsis TOR FRB domain could bind the yeast (Sormani et al., 2007) or human (Mahfouz etal., 2006) FKBP12 proteins in the presence of rapamycin. This opened the possibility of increasing plant sensitivity toward rapamycin by expressing the yeast FKBP12 protein (Sormani etal., 2007; Leiber et al., 2010; Ren et al., 2012). Conversely, the unicellular green alga Chlamydomonas is sensitive to moderate levels of rapamycin (100-500 nM), a concentration range similar to the one necessary to inhibit yeast growth (Heitman et al., 1991; Crespo et al., 2005). This can be explained by the fact that the algal FKBP12 protein is closer to human or yeast homologs and the residues critical for binding rapamycin are conserved only in Chlamydomonas. Nevertheless, it was recently shown that rapamycin, when added repeatedly at high concentrations $(1-10 \mu \mathrm{M})$ to liquid cultures of Arabidopsis, could affect plant growth and development (Xiong and Sheen, 2012).

This varying and reduced susceptibility of plants to rapamycin has clearly delayed the development of molecular studies on the plant TOR signaling pathway. Moreover the disruption of the AtTOR gene by T-DNA insertions was shown to be embryo lethal (Menand et al., 2002; Ren et al., 2011), which precluded the use of these mutants to further study the role of TOR in plants. To circumvent these difficulties we have produced constitutive and ethanol-inducible RNAi lines which allow a stable or conditional silencing of the AtTOR gene (Deprost et al., 2007). This study and other reports using estradiol-inducible artificial microRNA (amiRNA) showed that, when the expression of AtTOR was silenced, plant growth was arrested and several metabolites accumulated, including starch, triacylglycerides (TAGs), and amino acids (Deprost et al., 2007; Dobrenel et al., 2011; Xiong and Sheen, 2012; Caldana et al., 2013). This was accompanied by vast modifications in the plant transcriptome. Arabidopsis plants silenced for the AtTOR expression also displayed a significant reduction in polysome abundance (Deprost et al., 2007), in the phosphorylation of the ribosomal S6 kinase (S6K, Schepetilnikov et al., 2011; Xiong and Sheen, 2012) and were presenting signs of constitutive autophagy (Liu and Bassham, 2010). These results suggest that the main biological targets of the yeast and animal TORC1 complex, namely, S6K, mRNA translation, and autophagy are conserved during evolution. All these Arabidopsis lines provided invaluable tools to start deciphering the metabolic consequences of the inhibition of TOR activity in time-course experiments. It should be stressed that TOR inhibition by RNAi is likely to reveal a larger spectrum of phenotypes than rapamycin since this drug is known to inhibit only a subset of TORC1 activities, and not the TORC2 complex (Feldman et al., 2009; Guertin and Sabatini, 2009; Thoreen et al., 2009). Accordingly recent data suggest that knocking out TOR activity by silencing has more profound consequences than partly inhibiting the TORC1 complex with rapamycin (Ren et al., 2012).

\section{REGULATION OF THE TORC1 COMPLEX BY SUGARS}

In yeast it has been shown that carbon or nitrogen starvation inhibits TORC1 activity and that rapamycin action mimics the effects of nutrient removal by, for example, inducing autophagy or the expression of genes involved in the utilization of alternative source of nutrients (Rohde et al., 2008; Broach, 2012). It was for a long time unclear how nutrients regulated TORC1 activity, but recent reports nicely demonstrated that the vacuolar $\mathrm{H}^{+}$ATPase (v-ATPase) activates the TORC1 complex by recruiting it to the surface of yeast vacuoles or animal lysosomes in the presence of amino acids (Binda et al., 2009; Zoncu et al., 2011). This recruitment of TOR and the subsequent increase in TORC1 activity are mediated by the Rheb and Rag GTPase complexes (Cornu et al., 2013). Very recently it was found that glucose also induces TOR activity by regulating the binding of the v-ATPase to Rag GTPases, thus suggesting a shared regulatory mechanism between sugars and amino acids (Efeyan et al., 2013). Moreover the glycolytic enzyme glyceraldehyde-3-phosphate dehydrogenase (GAPDH) binds Rheb in low-glucose conditions and inhibits mTORC1 (mammalian target of rapamycin complex 1) signaling (Lee et al., 2009). Interestingly, in plants, the v-ATPase has also important roles in nutrient storage and signaling (Schumacher and Krebs, 2010). Similarly glucose, an important plant regulatory molecule, has been shown to be linked to TOR activation in Arabidopsis (Xiong and Sheen, 2012). The class III/Vps34 PI3K (phosphoinositide 3-kinase) has also been involved in nutrient activation of TORC1 through the production of PI3P (Gulati et al., 2008). Since this kinase is well-conserved in plants and affects the TOR signaling pathway (Turck et al., 2004), it would be quite interesting to evaluate its contribution to the nutrient regulation of TORC1.

\section{ROLE OF THE PLANT TORC1 COMPLEX IN STARCH AND RAFFINOSE ACCUMULATION}

Inhibition of TOR activity results in $\mathrm{C}$ storage through glycogen accumulation in animal muscles and yeast (Schmelzle et al., 2004; Cornu et al., 2013). Conversely TOR inhibition in the liver decreases the level of stored glycogen and the animals become hyperglycemic, a situation also found in type 2 diabetes. This suggests a prominent role of TOR in maintaining animal glucose homeostasis (Cornu et al., 2013). In yeast TORC1 inhibition by rapamycin triggers a switch from fermentation to respiration by reducing the expression of genes encoding glycolytic enzymes 
and increasing the expression of genes encoding tri carboxylic acid (TCA) enzymes (Figure 1A; De Virgilio and Loewith, 2006). The TORC1 signaling pathway is evidently important for cell metabolism and proliferation, therefore its perturbation is implicated in many human diseases (Cornu etal., 2013). Indeed activation of the TOR kinase is frequently encountered in human cancers and has been found to promote the flux of $\mathrm{C}$ through glycolysis by up-regulating genes involved in glucose uptake and glycolysis. TOR activation also stimulates lipogenesis and the pentose phosphate pathway (Düvel etal., 2010; Yecies and Manning, 2011).

In plants most of the stored C is found in starch. Several studies have established that starch is mainly synthesized during the day within the chloroplasts and degraded at night to provide cells with C metabolites and energy (for an excellent and recent review see Stitt and Zeeman, 2012). When limited in nitrogen, Chlamydomonas cells accumulate starch (Ball et al., 2011) and TAG (Siaut et al., 2011). Similarly, plants affected in nitrate assimilation accumulate starch at high levels (Saux et al., 1987). These results point clearly to a control of starch synthesis by the availability of nitrogen. Indeed in conditions where nitrogen is limiting, and growth is therefore reduced, most of the available $\mathrm{C}$ seems to be redirected toward the accumulation of reserves rather than toward other sinks like the synthesis of cell wall or the production of energy. Consistently, metabolite profiling of Arabidopsis accessions has shown that starch and sucrose contents are negatively correlated with rosette growth in Arabidopsis (Sulpice et al., 2009, 2010).

Conditional silencing of the Arabidopsis TOR gene led to a decrease in photosynthesis with a yellowing of the leaves linked to chlorophyll breakdown (Deprost et al., 2007). These symptoms of early senescence where accompanied by an accumulation of high amounts of soluble sugars, amino acids, and starch (Figure 1B). This suggests that TOR activity is needed to restrain senescence and could thus be involved in the regulation of life span in Arabidopsis (Deprost et al., 2007; Ren et al., 2012). A recent study also showed that TOR inhibition in Arabidopsis by inducible amiRNA results in high levels of starch accumulation (Figure 1B) together with increased levels of TAG (Caldana et al., 2013). A concomitant increase in TCA cycle intermediates was also detected after TOR inhibition by either amiRNA (Caldana et al., 2013), the treatment by rapamycin of Arabidopsis lines expressing a FKBP12 protein (Ren et al., 2012) or in lst8 mutants (Moreau et al., 2012). The same perturbation in the TCA cycle was observed in yeast treated with rapamycin (De Virgilio and Loewith, 2006).

In the study by Sulpice etal. (2009) described above, it was found that rosette biomass negatively correlated with the amount of starch but a strong positive correlation was detected with the expression of myo-inositol-1-phosphate synthase 1 (MIPS1/At4g39800). MIPS is conserved in all eukaryotes and catalyzes the first committed step in the synthesis of myo-inositol, a central C-metabolite that serves in the synthesis of the signaling lipids (phosphatidyl)inositol-phosphate (PIP), of cell wall precursors like UDP-glucuronate and of the raffinose family oligosaccharides (RFOs, Figure 2; for a review see Valluru and Van den Ende, 2011). The levels of raffinose, of its precursor galactinol and of myo-inositol, which serves as a cofactor in this biosynthetic pathway, are often strongly correlated (Figure 2; G.

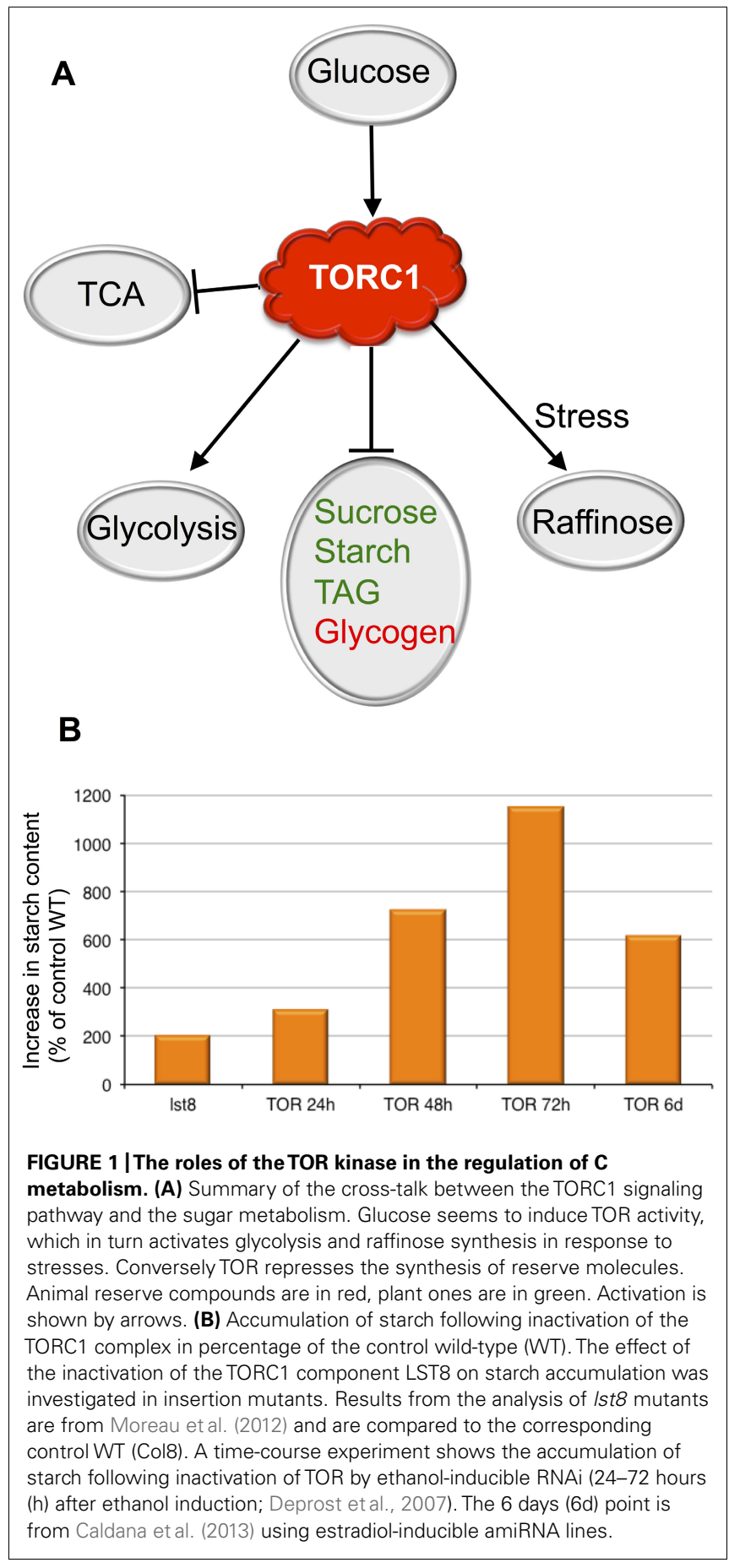

Clément, personal communication; Sulpice et al., 2010). A survey of metabolite profiling experiments shows that raffinose accumulates in stress situations like high light, nitrogen starvation, or high salt (G. Clément, personal communication and unpublished data).

A common trend that emerges from the analysis of stressed Arabidopsis plants affected in the activity of the TORC1 complex is the decrease in raffinose and galactinol accumulation. Ren et al. (2012) observed lower levels of raffinose and myo-inositol 


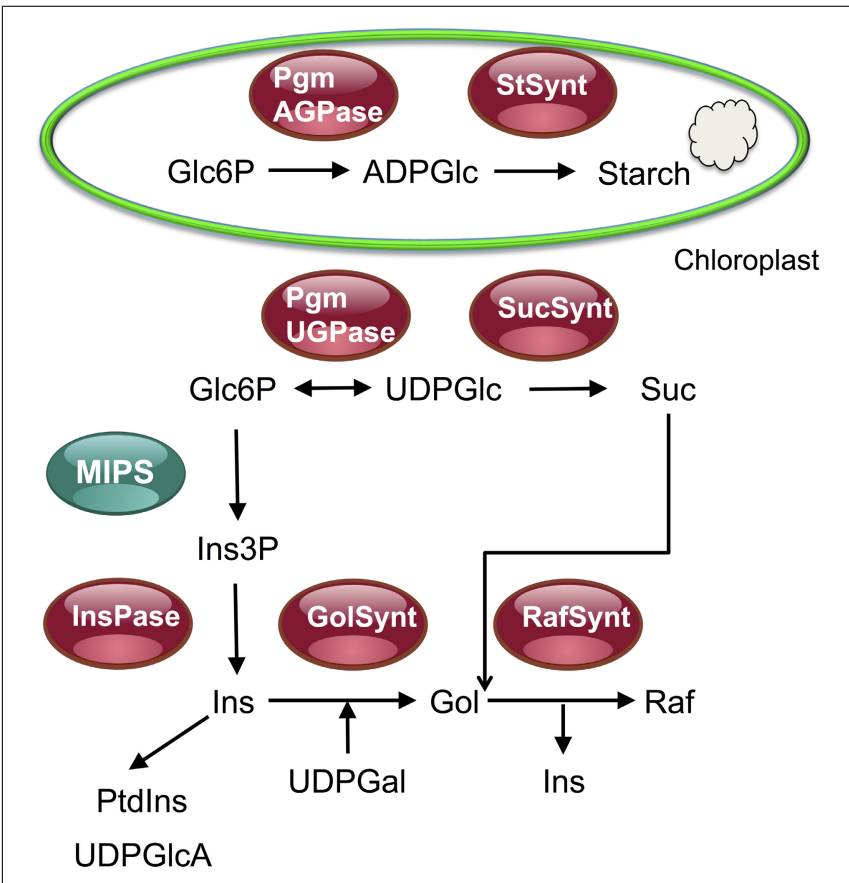

FIGURE 2 | Overview of the raffinose synthesis pathway and of its relationship with the sucrose and starch synthesis pathways. This figure shows that glucose 6-phosphate (GIc6P) can be used either for the synthesis of starch in chloroplasts or for the synthesis of sucrose or myo-inositol and raffinose in the cytosol. After TOR inactivation, or mutations in the TORC1 components RAPTOR and LST8, an accumulation of starch and sucrose was observed together with a decrease in the synthesis of raffinose in stress conditions. This suggests TOR inactivation triggers a redirection of $\mathrm{C}$ fluxes toward starch and sucrose for, respectively, storage and export. Pgm, phosphoglucomutase; StSynt, starch synthase; AGPase, ADPglucose pyrophosphorylase; UGPase, UDPglucose pyrophosphorylase; SucSynt, sucrose synthase; MIPS, myo-inositol 3-phosphate synthase; Ins(3P), myo-inositol (3 phosphate): InsPase, inositol monophosphate phosphatase; Ptdlns,

phosphatidylinositol; UDPGIcA, UDP-glucuronic acid; Gol(Synt), galactinol (synthase); Raf(Synt), raffinose (synthase).
Like lst8 mutants (Moreau et al., 2012), plants affected in the MIPS1 gene expression were described as lacking galactinol accumulation and being sensitive to long days (Meng et al., 2009). Accordingly the transcriptome variations due to either mips1 or lst 8 mutations shared some striking similarities. Indeed, nearly $70 \%$ of the genes differentially expressed in the $l s t 8$ mutant when shifted to long days were also found to be either down- or upregulated in the mips1 mutant (Moreau et al., 2012). This indicates that a large proportion of the impact of $l s t 8$ mutations, which probably result in a decreased TORC1 activity, on the adaptation to long days can be explained by a default in MIPS1 activity and thus by a decrease in myo-inositol production. This default in raffinose and galactinol production after extension of the light period could explain the arrest of growth and the severe phenotype of both $l s t 8$ and mips 1 mutants following a shift to long day conditions.

Furthermore MIPS and galactinol synthases were found to be significantly repressed in TOR RNAi lines or in the $1 s t 8$ mutants grown in long days. These results are in agreement with the observed lack of galactinol and raffinose accumulation in plants where the TORC1 activity is reduced (Moreau et al., 2012; Ren et al., 2012). The MIPS genes could therefore serve as a hub for adjusting the plant metabolism to changes in environmental conditions. The inducible overexpression of the bZIP11 transcription factor, which is normally up-regulated by the SnRK1 kinase, results in an augmented level of raffinose (Ma et al., 2011). This is consistent with the fact that raffinose is also accumulated in response to multiple stresses (Valluru and Van den Ende, 2011). Indeed this could possibly be the result of the activation of the SnRK1 kinase in stress conditions (Robaglia et al., 2012). Nevertheless it is surprising, given the expected opposite role of the TOR and SnRK1 kinases (Robaglia et al., 2012), that TOR also seems to be required for raffinose production. One explanation could be that TORC1 activity is also needed for bZIP11 expression and it would be interesting to determine if the bZIP11-induced accumulation of raffinose is TOR-dependent.

\section{CONCLUSION}

It is now clear that inhibiting the TORC1 activity results in starch and TAG accumulation (Dobrenel et al., 2011; Caldana et al., 2013), a decrease in biomass production but also a decrease in protein concentration and mRNA translation (Deprost et al., 2007; Sormani etal., 2007; Ren etal., 2012; Xiong and Sheen, 2012; Caldana et al., 2013). It thus appears that the TOR signaling pathway may contribute to the close link between starch, protein, and biomass observed in plants (Sulpice et al., 2009, 2010). The signals triggering starch accumulation and re-routing of $\mathrm{C}$ fluxes in response to TORC1 inactivation remain to be determined, but it is striking that the accumulation of starch observed in TORC1-deficient Arabidopsis plants is accompanied by a decrease in raffinose production, both being dependent on the supply of glucose-6P (Figure 2). The TORC1 activity is probably also required for plant adaptation to stresses by stimulating synthesis of myo-inositol and RFOs. Whether they serve as $\mathrm{C}$ storage molecules or for the scavenging of reactive oxygen species remains to be determined more clearly. 
Since the inactivation of TOR results, as in other eukaryotes, in the accumulation of reserve molecules in plants (TAG and starch), it can be anticipated that the regulation of TOR activity in developing seeds may also be of importance for the synthesis of seed storage compounds. Moreover, using TOR inactivation to redirect C fluxes toward reserves compounds like starch or TAG, which are easier to process than lignocellulosic molecules, could foster the use of plants for the production of biofuels and other bio-based components.

\section{REFERENCES}

Anderson, G., Veit, B., and Hanson, M. (2005). The Arabidopsis AtRaptor genes are essential for postembryonic plant growth. BMC Biol. 3:12. doi: 10.1186/1741-7007-3-12

Ball, S., Colleoni, C., Cenci, U., Raj, J. N., and Tirtiaux, C. (2011). The evolution of glycogen and starch metabolism in eukaryotes gives molecular clues to understand the establishment of plastid endosymbiosis. J. Exp. Bot. 62, 1775-1801.

Binda, M., Peli-Gulli, M. P., Bonfils, G., Panchaud, N., Urban, J., Sturgill, T. W., et al. (2009). The Vam6 GEF controls TORC1 by activating the EGO complex. Mol. Cell 35, 563-573.

Broach, J. R. (2012). Nutritional control of growth and development in yeast. Genetics 192, 73-105.

Caldana, C., Li, Y., Leisse, A., Zhang, Y., Bartholomaeus, L., Fernie, A. R., et al. (2013). Systemic analysis of inducible target of rapamycin mutants reveal a general metabolic switch controlling growth in Arabidopsis thaliana. Plant J. 73, 897-909.

Cornu, M., Albert, V., and Hall, M. N. (2013). mTOR in aging, metabolism, and cancer. Curr. Opin. Genet. Dev. doi: 10.1016/j.gde.2012.12.005 [Epub ahead of print].

Crespo, J., Díaz-Troya, S., and Florencio, F. (2005). Inhibition of target of rapamycin signaling by rapamycin in the unicellular green alga Chlamydomonas reinhardtii. Plant Physiol. 139, 1736-1749.

Deprost, D., Truong, H., Robaglia, C., and Meyer, C. (2005). An Arabidopsis homolog of RAPTOR/KOG1 is essential for early embryo development. Biochem. Biophys. Res. Commun. 326 , 844-850.

Deprost, D., Yao, L., Sormani, R., Moreau, M., Leterreux, G., Nicolai, M., et al. (2007). The Arabidopsis TOR kinase links plant growth, yield, stress resistance and mRNA translation. EMBO Rep. 8, 864-870.

De Virgilio, C., and Loewith, R. (2006). The TOR signalling network from yeast to man. Int. J. Biochem. Cell Biol. 38, 1476-1481.

Díaz-Troya, S., Florencio, F., and Crespo, J. (2008). Target of rapamycin and LST8 proteins associate with membranes from the endoplasmic reticulum in the unicellular green alga Chlamydomonas reinhardtii. Eukaryot. Cell 7, 212-222.

Dobrenel, T., Marchive, C., Sormani, R., Moreau, M., Mozzo, M., Montané, M. H., et al. (2011). Regulation of plant growth and metabolism by the TOR kinase. Biochem. Soc. Trans. 39, 477-481.

Düvel, K., Yecies, J. L., Menon, S., Raman, P., Lipovsky, A. I., Souza, A. L., et al. (2010). Activation of a metabolic gene regulatory network downstream of mTOR complex 1 . Mol. Cell 39, 171-183.

Efeyan, A., Zoncu, R., Chang, S., Gumper, I., Snitkin, H., Wolfson, R. L., et al. (2013). Regulation of mTORC1 by the Rag GTPases is necessary for neonatal autophagy and survival. Nature 493, 679-683.

Feldman, M. E., Apsel, B., Uotila, A., Loewith, R., Knight, Z. A., Ruggero, D., et al. (2009). Active-site inhibitors of mTOR target rapamycin-resistant outputs of mTORC1 and mTORC2. PLoS Biol. 7:e38. doi: 10.1371/journal.pbio. 1000038

Guertin, D. A., and Sabatini, D. M. (2009). The pharmacology of mTOR inhibition. Sci. Signal. 2, pe24.

Gulati, P., Gaspers, L. D., Dann, S. G., Joaquin, M., Nobukuni, T., Natt, F., et al. (2008). Amino acids activate mTOR complex 1 via Ca2+/CaM signaling to hVps34. Cell Metab. 7, 456-465.

Heitman, J., Movva, N. R., and Hall, M. N. (1991). Targets for cell cycle arrest by the immunosuppressant rapamycin in yeast. Science 253, 905-909.

John, F., Roffler, S., Wicker, T., and Ringli, C. (2011). Plant TOR signaling components. Plant Signal. Behav. 6, 1700-1705.

Keunen, E., Peshev, D., Vangronsveld, J., Van den Ende, W., and Cuypers, A. (2013). Plant sugars are crucial players in the oxidative challenge during abiotic stress. Extending the traditional concept. Plant Cell Environ. doi: 10.1111/pce.12061 [Epub ahead of print].

\section{ACKNOWLEDGMENTS}

This work was partly supported by ANR grants (ANR Blanc063-135436 and Blanc2011-SV6-01002) to Christian Meyer, Rodnay Sormani, and Christophe Robaglia. Manon Moreau was supported by a joint PhD grant from INRA (Plant Biology Department) and DSV CEA. Chloé Marchive was supported by a joint INRA-FAPESP grant. We thank our colleagues for fruitful discussions and the reviewers for improving our manuscript.

Laplante, M., and Sabatini, D. M. (2012). mTOR signaling in growth control and disease. Cell 149, 274-293.

Lee, M. N., Ha, S. H., Kim, J., Koh, A., Lee, C. S., Kim, J. H., et al. (2009). Glycolytic flux signals to mTOR through glyceraldehyde-3phosphate dehydrogenase-mediated regulation of Rheb. Mol. Cell. Biol. 29, 3991-4001.

Leiber, R., John, F., Verhertbruggen, Y., Diet, A., Knox, J., and Ringli, C. (2010). The TOR pathway modulates the structure of cell walls in Arabidopsis. Plant Cell 22, 1898-1908.

Liu, Y., and Bassham, D. (2010). TOR is a negative regulator of autophagy in Arabidopsis thaliana. PLoS ONE 5:e11883. doi: 10.1371/journal.pone.0011883

Loewith, R., and Hall, M. N. (2011). Target of rapamycin (TOR) in nutrient signaling and growth control. Genetics 189, 1177-1201.

Ma, J., Hanssen, M., Lundgren, K., Hernandez, L., Delatte, T., Ehlert, A., et al. (2011). The sucrose-regulated Arabidopsis transcription factor bZIP11 reprograms metabolism and regulates trehalose metabolism. New Phy tol. 191, 733-745.

Ma, X. M., and Blenis, J. (2009). Molecular mechanisms of mTOR-mediated translational control. Nat. Rev. Mol. Cell Biol. 10, 307-318.

Mahfouz, M., Kim, S., Delauney, A., and Verma, D. (2006). Arabidopsis TARGET OF RAPAMYCIN interacts with RAPTOR, which regulates the activity of S6 kinase in response to osmotic stress signals. Plant Cell 18, 477-490.

Menand, B., Desnos, T., Nussaume, L., Berger, F., Bouchez, D., Meyer, C., et al. (2002). Expression and disruption of the Arabidopsis TOR (target of rapamycin) gene. Proc. Natl. Acad. Sci. U.S.A. 99, 6422-6427.

Meng, P. H., Raynaud, C., Tcherkez, G., Blanchet, S., Massoud, K., Domenichini, S., et al. (2009). Crosstalks between myo-inositol metabolism, programmed cell death and basal immunity in Arabidopsis. PLoS ONE 4:e7364. doi: 10.1371/journal.pone. 0007364
Moreau, M., Azzopardi, M., Clément, G., Dobrenel, T., Marchive, C., Renne, C., et al. (2012). Mutations in the Arabidopsis homolog of LST8/G $\beta$ L, a partner of the target of Rapamycin kinase, impair plant growth, flowering, and metabolic adaptation to long days. Plant Cell 24, 463-481.

Nishizawa, A., Yabuta, Y., and Shigeoka, S. (2008). Galactinol and raffinose constitute a novel function to protect plants from oxidative damage. Plant Physiol. 147, 1251-1263.

Peters, S., Egert, A., Stieger, B., and Keller, F. (2010). Functional identification of Arabidopsis ATSIP2 (At3g57520) as an alkaline alphagalactosidase with a substrate specificity for raffinose and an apparent sink-specific expression pattern. Plant Cell Physiol. 51, 1815-1819.

Ren, M., Qiu, S., Venglat, P., Xiang, D., Feng, L., Selvaraj, G., et al. (2011). Target of rapamycin regulates development and ribosomal RNA expression through kinase domain in Arabidopsis. Plant Physiol. 155, 1367-1382.

Ren, M., Venglat, P., Qiu, S., Feng, L., Cao, Y., Wang, E., et al. (2012). Target of rapamycin signaling regulates metabolism, growth, and life span in Arabidopsis. Plant Cell 24, 4850-4874.

Robaglia, C., Thomas, M., and Meyer, C. (2012). Sensing nutrient and energy status by SnRK1 and TOR kinases. Curr. Opin. Plant Biol. 15, 301-307.

Rohde, J. R., Bastidas, R., Puria, R., and Cardenas, M. E. (2008). Nutritional control via Tor signaling in Saccharomyces cerevisiae. Curr. Opin. Microbiol. 11, 153-160.

Saux, C., Lemoine, Y., Marion-Poll, A., Valadier, M. H., Deng, M., and Morot-Gaudry, J. F. (1987). Consequence of absence of nitrate reductase activity on photosynthesis in Nicotiana plumbaginifolia plants. Plant Physiol. 84, 67-72.

Schepetilnikov, M., Kobayashi, K., Geldreich, A., Caranta, C., Robaglia, C., Keller, M., et al. (2011). Viral factor TAV recruits TOR/S6K1 signalling to activate reinitiation after long ORF translation. EMBO J. 6, 1343-1356.

Schmelzle, T., Beck, T., Martin, D. E., and Hall, M. N. (2004). Activation 
of the RAS/cyclic AMP pathway suppresses a TOR deficiency in yeast. Mol. Cell. Biol. 24, 338-351.

Schumacher, K., and Krebs, M. (2010). The V-ATPase: small cargo, large effects. Curr. Opin. Plant Biol 13, 724-730.

Siaut, M., Cuine, S., Cagnon, C., Fessler, B., Nguyen, M., Carrier, P., et al. (2011). Oil accumulation in the model green alga Chlamydomonas reinhardtii: characterization, variability between common laboratory strains and relationship with starch reserves. BMC Biotechnol. 11:7. doi: 10.1186/1472-6750-11-7

Sormani, R., Yao, L., Menand, B., Ennar, N., Lecampion, C., Meyer, C., et al. (2007). Saccharomyces cerevisiae FKBP12 binds Arabidopsis thaliana TOR and its expression in plants leads to rapamycin susceptibility. $B M C$ Plant Biol. 7:26. doi: 10.1186/14712229-7-26

Stitt, M., and Zeeman, S. C. (2012). Starch turnover: pathways, regulation and role in growth. Curr. Opin. Plant Biol. 15, 282-292.

Sulpice, R., Pyl, E. T., Ishihara, H., Trenkamp, S., Steinfath, M., WituckaWall, H., etal. (2009). Starch as a major integrator in the regulation of plant growth. Proc. Natl.
Acad. Sci. U.S.A. 106, 10348 10353.

Sulpice, R., Trenkamp, S., Steinfath, M., Usadel, B., Gibon, Y., Witucka-Wall, H., et al. (2010). Network analysis of enzyme activities and metabolite levels and their relationship to biomass in a large panel of Arabidopsis accessions. Plant Cell 22, 2872-2893.

Taji, T., Ohsumi, C., Iuchi, S., Seki, M., Kasuga, M., Kobayashi, M., et al. (2002). Important roles of droughtand cold-inducible genes for galactinol synthase in stress tolerance in Arabidopsis thaliana. Plant J. 29, 417-426.

Thoreen, C. C., Kang, S. A., Chang, J. W., Liu, Q., Zhang, J., Gao, Y., et al. (2009). An ATP-competitive mammalian target of rapamycin inhibitor reveals rapamycin-resistant functions of mTORC1. J. Biol. Chem. 284 8023-8032.

Turck, F., Zilbermann, F., Kozma, S., Thomas, G., and Nagy, F. (2004). Phytohormones participate in an S6 kinase signal transduction pathway in Arabidopsis. Plant Physiol. 134, 1527-1535.

Valluru, R., and Van den Ende, W. (2011). Myo-inositol and beyond-emerging networks under stress. Plant Sci. 181, 387400.

Wang, X., and Proud, C. G. (2011). mTORC1 signaling: what we still don't know. J. Mol. Cell Biol. 3, 206-220.

Wullschleger, S., Loewith, R., and Hall, M. (2006). TOR signaling in growth and metabolism. Cell 124, 471-484.

Xiong, Y., and Sheen, J. (2012). Rapamycin and glucose-target of rapamycin (TOR) protein signaling in plants. J. Biol. Chem. 287, 2836-2842.

$\mathrm{Xu}$, Q., Liang, S., Kudla, J., and Luan, S. (1998). Molecular characterization of a plant FKBP12 that does not mediate action of FK506 and rapamycin. Plant J. 15, 511-519.

Yecies, J. L., and Manning, B. D. (2011). Transcriptional control of cellular metabolism by mTOR signaling. Cancer Res. 71, 2815-2820.

Zoncu, R., Bar-Peled, L., Efeyan, A., Wang, S., Sancak, Y., and Sabatini, D. M. (2011). mTORC1 senses lysosomal amino acids through an inside-out mechanism that requires the vacuolar $\mathrm{H}^{+}$-ATPase. Science 334, 678-683.
Conflict of Interest Statement: The authors declare that the research was conducted in the absence of any commercial or financial relationships that could be construed as a potential conflict of interest.

Received: 06 February 2013; paper pending published: 21 February 2013; accepted: 27 March 2013; published online: 15 April 2013.

Citation: Dobrenel T, Marchive C, Azzopardi M, Clément G, Moreau M, Sormani $R$, Robaglia $C$ and Meyer $C$ (2013) Sugar metabolism and the plant target of rapamycin kinase: a sweet operaTOR? Front. Plant Sci. 4:93. doi: 10.3389/fpls.2013.00093

This article was submitted to Frontiers in Plant Physiology, a specialty of Frontiers in Plant Science.

Copyright $\odot 2013$ Dobrenel, Marchive, Azzopardi, Clément, Moreau, Sormani, Robaglia and Meyer. This is an openaccess article distributed under the terms of the Creative Commons Attribution License, which permits use, distribution and reproduction in other forums, provided the original authors and source are credited and subject to any copyright notices concerning any third-party graphics etc. 